\title{
On Spatial Asymmetric Games
}

\author{
E. Ahmed ${ }^{1,2}$, A. S. Hegazi ${ }^{1,2}$ and A. S. Elgazzar ${ }^{3}$ \\ ${ }^{1}$ Mathematics Department, Faculty of Science \\ 35516 Mansoura, Egypt \\ ${ }^{2}$ Mathematics Department, Faculty of Science \\ Al-Ain PO Box 17551, UAE \\ ${ }^{3}$ Mathematics Department, Faculty of Education \\ 45111 El-Arish, Egypt
}

\begin{abstract}
The stability of some spatial asymmetric games is discussed. Both linear and nonlinear asymptotic stability of asymmetric hawk-dove and prisoner's dilemma are studied. Telegraph reaction diffusion equations for the asymmetric spatial games are presented. Asymmetric game of parental investment is studied in the presence of both ordinary and cross diffusions.
\end{abstract}

\section{Introduction}

In asymmetric games [Hofbauer and Sigmund 1998], different players have different strategies and different payoffs. In reality, most games are asymmetric e.g. battle of the sexes and owners-intruders games. The differential equations of the asymmetrical games are

$$
\begin{aligned}
\frac{\mathrm{d} p_{i}}{\mathrm{~d} t} & =p_{i}\left[(A q)_{i}-p A q\right] \\
\frac{\mathrm{d} q_{i}}{\mathrm{~d} t} & =q_{i}\left[(B p)_{i}-q B p\right]
\end{aligned}, i=1,2, \ldots, n,
$$

where $A(B)$ is the payoff matrix of the first (second) player, and $p_{i}\left(q_{i}\right)$ is the fraction of adopters of the strategy $i$ in the first (second) population, respectively. 
The problem of Turing instability (diffusion induced instability) [Okubo 1980] for symmetric games have been already studied [Cressman and Vickers 1997]. In this case, the standard replicator equation for the symmetric game is given by

$$
\frac{\partial p_{i}}{\partial t}=p_{i}\left[(A p)_{i}-p A p\right]+D \nabla^{2} p_{i}
$$

It is known [Okubo 1980] that including spatial effects may significantly change the stability of equilibrium points. Also spatial effects are crucial in many biological phenomena. Some authors [Boerlijst and Hogeweg 1995] have studied general spatial hypercycle systems. Therefore studying spatial asymmetric games is an important problem. Due to the difficulty in defining evolutionarily stable strategy (ESS) in asymmetric games [Hofbauer and Sigmund 1998], we will concentrate on asymptotically stable strategies. The equations of spatial asymmetric games are [Hofbauer et al 1997]

$$
\begin{aligned}
& \frac{\partial p_{i}}{\partial t}=p_{i}\left[(A q)_{i}-p A q\right]+D_{1} \nabla^{2} p_{i}, \\
& \frac{\partial q_{i}}{\partial t}=q_{i}\left[(B p)_{i}-q B p\right]+D_{2} \nabla^{2} q_{i} .
\end{aligned}
$$

In this paper, we will attempt to answer the following questions:

1. Given an asymptotically stable solution to the system (1), does Turing instability exist for the corresponding spatial game (3)?

2. Given an asymptotically unstable solution to the system (1), can diffusion stabilize it?

3. Given an asymptotically linearly stable solution to the system (1), is it nonlinearly stable?

Our typical examples will be the asymmetric hawk-dove (AHD) and the asymmetric prisoner's dilemma (APD) games.

The paper is organized as follows: In section 2, the asymmetric hawkdove game is studied. Conditions for Turing stability and nonlinear finite amplitude instability are derived. In section 3, The asymmetric prisoner's dilemma is presented. Telegraph reaction diffusion equation is applied for stability analysis of the asymmetric prisoner's dilemma game in section 4 . In section 5, an asymmetric game of parental investment will be studied. Some conclusions are summarized in section 6 . 


\section{Asymmetric hawk-dove game}

In this case the possible strategies are hawk $(\mathrm{H})$ and dove $(\mathrm{D})$, and the payoff matrices $A$ and $B$ in Eq. (1) become

$$
A=\left[\begin{array}{cc}
\frac{1}{2}\left(v_{1}-c_{1}\right) & v_{1} \\
0 & \frac{v_{1}}{2}
\end{array}\right], B=\left[\begin{array}{cc}
\frac{1}{2}\left(v_{2}-c_{2}\right) & v_{2} \\
0 & \frac{v_{2}}{2}
\end{array}\right],
$$

where $c_{i}>v_{i}, i=1,2$. The corresponding partial differential equations for the spatial AHD are

$$
\begin{aligned}
& \frac{\partial p}{\partial t}=D_{1} \nabla^{2} p+\frac{1}{2} p(1-p)\left(v_{1}-c_{1} q\right), \\
& \frac{\partial q}{\partial t}=D_{2} \nabla^{2} q+\frac{1}{2} q(1-q)\left(v_{2}-c_{2} p\right),
\end{aligned}
$$

where $p(q)$ is the fraction of hawks in the population of the first (second) player. It is direct to see that the solution $p=1, q=0$ is linearly asymptotically stable solution for the system (5), without diffusion $\left(D_{1}=D_{2}=0\right)$.

The first question is about Turing (diffusion induced) instability [Okubo 1980]. It occurs if the following system

$$
\begin{aligned}
& \frac{\partial p}{\partial t}=D_{1} \frac{\partial^{2} p}{\partial x^{2}}+f_{1}(p, q), \\
& \frac{\partial q}{\partial t}=D_{2} \frac{\partial^{2} q}{\partial x^{2}}+f_{2}(p, q),
\end{aligned}
$$

has an equilibrium solution $\left(p_{s s}, q_{s s}\right)$ which is stable if $D_{1}=D_{2}=0$, and the corresponding linearized system

$$
\begin{gathered}
p=p_{s s}+\varepsilon(x, t), q=q_{s s}+\eta(x, t), \\
\frac{\partial \varepsilon}{\partial t}=D_{1} \frac{\partial^{2} \varepsilon}{\partial x^{2}}+a_{11} \varepsilon+a_{12} \eta, \\
\frac{\partial \eta}{\partial t}=D_{2} \frac{\partial^{2} \eta}{\partial x^{2}}+a_{21} \varepsilon+a_{22} \eta,
\end{gathered}
$$

satisfies the condition

$$
H\left(k^{2}\right)=D_{1} D_{2} k^{4}-\left(D_{1} a_{22}+D_{2} a_{11}\right) k^{2}+a_{11} a_{22}-a_{12} a_{21}<0
$$

In this case diffusion will destabilize the solution $\left(p_{s s}, q_{s s}\right)$. This is Turing instability.

Applying the above procedure to the spatial AHD one gets:

Proposition (1): The equilibrium solution $p=1, q=0$ of the AHD game is Turing stable. 
The second question to be discussed is: Can diffusion stabilize an unstable solution of the AHD game? Consider the internal solution $p=v_{2} / c_{2}, q=$ $v_{1} / c_{1}$. It is asymptotically unstable solution to the diffusionless case $\left(D_{1}=\right.$ $\left.D_{2}=0\right)$. Including diffusion and linearizing around $p=v_{2} / c_{2}, q=v_{1} / c_{1}$, and assuming the following boundary conditions:

$$
\begin{gathered}
p=\frac{v_{2}}{c_{2}}+\varepsilon(x, t), q=\frac{v_{1}}{c_{1}}+\eta(x, t), \\
\varepsilon(0, t)=\varepsilon(1, t)=0, \eta(0, t)=\eta(1, t)=0,
\end{gathered}
$$

then

Proposition (2): The interior solution $p=v_{2} / c_{2}, q=v_{1} / c_{1}$, with the boundary conditions (9) is linearly asymptotically stable if

$$
D_{1} D_{2} \pi^{4}-\frac{v_{1} v_{2}}{4}\left(1-\frac{v_{1}}{c_{1}}\right)\left(1-\frac{v_{2}}{c_{2}}\right) \geq 0 .
$$

Linear stability analysis is useful if the perturbations of equilibrium are infinitesimally small. This is not always the case in biological systems. In this case one has to study finite amplitude instability (FAI) [Stuart 1989] of the equilibrium solution. In the following, we generalize the work of Stuart to the two species case. Consider the following equation

$$
\frac{\partial \theta}{\partial t}=\frac{\partial^{2} \theta}{\partial x^{2}}+f(\theta), \theta(0, t)=\theta(1, t)=0 .
$$

Linearizing around the solution $\theta=0$, i.e. let

$$
\theta(x, t)=v(x, t),
$$

linearize in $v$, then

$$
\begin{gathered}
\frac{\partial v}{\partial t}=\frac{\partial^{2} v}{\partial x^{2}}+v f^{\prime}(0), \text { Let } v=\phi(x) \exp (\sigma t) \Rightarrow \\
\sigma \phi=\phi^{\prime \prime}+f^{\prime}(0) \phi, \phi(0)=\phi(1)=0 .
\end{gathered}
$$

Set $\phi=\sum_{l} a_{l} \sin (\pi l x)$, then bifurcation points are given by $f^{\prime}(0)=(\pi l)^{2}, l=$ $1,2, \ldots$. Studying the stability of the first bifurcation point $l=1$ using Matkowsky two-time nonlinear stability analysis, one defines $\lambda=a-k$, set $\lambda=\pi^{2}+\lambda_{0} \varepsilon^{2}, \varepsilon$ is a small parameter. Decompose the time into fast $t^{\prime}$ and slow $\tau$, then 


$$
\frac{\partial}{\partial t}=\frac{\partial}{\partial t^{\prime}}+\varepsilon^{2} \frac{\partial}{\partial \tau}
$$

Expand $u$ in powers of $\varepsilon^{2}$, then $u \simeq \varepsilon v_{1}+\varepsilon^{3} v_{3}+\ldots$, (notice that $f^{\prime \prime}(0)=0$ ), then substituting in Eq. (12), one gets

$$
v_{1}\left(x, t^{\prime}, \tau\right)=\sum_{l} a_{l}(\tau) \sin (\pi l x) \exp \left(1-l^{2}\right) \pi^{2} t^{\prime} .
$$

Substituting into the cubic term and setting the constant term in $t^{\prime}$ equal to zero, one finally gets

$$
\begin{gathered}
\frac{d a_{1}}{d \tau}=\lambda_{0} a_{1}+b\left(a_{1}\right)^{3}, \\
b=4 \pi^{4} f^{\prime}(0) \frac{\int_{0}^{1} \sin ^{4} \pi x \mathrm{~d} x}{6 \int_{0}^{1} \sin ^{2} \pi x \mathrm{~d} x} .
\end{gathered}
$$

Thus a nonlinear (finite amplitude) instability arises if

$$
\lambda_{0}<0 \text { and }\left|a_{1}(0)\right|>\frac{\sqrt{\left|\lambda_{0}\right|}}{b} .
$$

In the beginning of the section the condition $f^{\prime \prime}(0)=0$ was imposed, here we will assume $f^{\prime \prime}(0) \neq 0$. Thus we consider

$$
\frac{\partial u}{\partial t}=D \nabla^{2} u+f(u), f(0)=0, u(0, t)=u(1, t)=0 .
$$

The solution $u=0$ is a steady state solution, so expanding near it we set

$$
u=\sum_{m=1} \varepsilon^{m} v_{m}\left(t^{\prime}, t^{\prime \prime}, x\right), \frac{\partial}{\partial t}=\frac{\partial}{\partial t^{\prime}}+\varepsilon \frac{\partial}{\partial t^{\prime \prime}}, f^{\prime}(0)=\lambda_{0}+\varepsilon \lambda_{1} .
$$

Substituting in Eq. (14) and equating terms $O(\varepsilon)$, one gets

$$
v_{1}\left(t^{\prime}, t^{\prime \prime}, x\right)=\sum_{l=1} a_{l}\left(t^{\prime \prime}\right) \sin (\pi l x) \exp \left[\left(1-l^{2}\right) \pi^{2}\right] .
$$

Let $\lambda_{0}=\pi$, and consider the equation $O\left(\varepsilon^{2}\right)$, we set the secular term (independent of $t^{\prime}$ ) equal to zero, then

$$
\frac{\mathrm{d} a_{1}}{\mathrm{~d} t^{\prime \prime}}=\lambda_{1} a_{1}+\left(\frac{b^{\prime}}{2} f^{\prime \prime}(0)\right) a_{1}^{2}, b^{\prime}=\frac{\int_{0}^{1} \sin ^{3} \pi x \mathrm{~d} x}{\int_{0}^{1} \sin ^{2} \pi x \mathrm{~d} x} .
$$


Thus the conditions for FAI are

$$
f^{\prime \prime}(0)>0, \lambda_{1}<0 \text { and } a_{1}(0)>-\frac{2 \lambda_{1}}{b^{\prime} f^{\prime \prime}(0)} .
$$

For systems of two partial differential equations

$$
\begin{gathered}
\frac{\partial u_{1}}{\partial t}=D_{1} \nabla^{2} u_{1}+f\left(u_{1}, u_{2}\right), \frac{\partial u_{2}}{\partial t}=D_{2} \nabla^{2} u_{2}+g\left(u_{1}, u_{2}\right), \\
u_{1}(0, t)=u_{1}(1, t)=u_{2}(0, t)=u_{2}(1, t)=0, \\
f(0,0)=g(0,0)=0 .
\end{gathered}
$$

Expanding near the steady state solutions $u_{1}=u_{2}=0$, we get

$$
\begin{gathered}
u_{1}=\sum_{m=1} \varepsilon^{m} v_{m}\left(t^{\prime}, t^{\prime \prime}, x\right), u_{2}=\sum_{m=1} \varepsilon^{m} \omega_{m}\left(t^{\prime}, t^{\prime \prime}, x\right), \\
\frac{\partial}{\partial t}=\frac{\partial}{\partial t^{\prime}}+\varepsilon \frac{\partial}{\partial t^{\prime \prime}}, \\
f_{1}=\frac{\partial f}{\partial u_{1}(0,0)}=\lambda_{11}+\varepsilon \lambda_{12}, f_{2}=\lambda_{21}+\varepsilon \lambda_{22}, \\
g_{1}=\mu_{11}+\varepsilon \mu_{12}, g_{2}=\mu_{21}+\varepsilon \mu_{22} .
\end{gathered}
$$

After some tedious calculations, we got the following conditions for FAI in the system (16):

(i) $\left(\pi^{2} D_{1}-\lambda_{11}\right)\left(\pi^{2} D_{2}-\mu_{21}\right)-\lambda_{21} \mu_{11}=0$.

(ii) $\lambda_{12}+\kappa \lambda_{22}=\frac{\mu_{12}}{\kappa}+\mu_{22}<0$, where $\kappa=\frac{\pi^{2} D_{1}-\lambda_{11}}{\lambda_{21}}$.

(iii) $\frac{f_{11}}{2}+\kappa f_{12}+\frac{\kappa^{2}}{2} f_{22}=\frac{g_{11}}{2 \kappa}+g_{12}+\frac{\kappa}{2} g_{22}>0$.

(iv) $a_{1}(0)>-\frac{\lambda_{12}+\kappa \lambda_{22}}{b^{\prime}\left(\frac{f_{11}}{2}+\kappa f_{12}+\frac{\kappa^{2}}{2} f_{22}\right)}$,

where $b^{\prime}$ is defined in Eq. (15). Applying the condition (i) to the spatial AHD, one gets

$$
\left(\pi^{2} D_{1}+v_{1}\right)\left(\pi^{2} D_{2}+v_{2}\right)<0,
$$

which is not possible, thus we find:

Proposition (3): There is no finite amplitude instability for the solution $p=1, q=0$ of the AHD game. 


\section{Asymmetric prisoner's dilemma game}

In the prisoner's dilemma game, the possible strategies are cooperate $(\mathrm{C})$ and defect (D). The payoff matrices $A, B$ in Eq. (1) are given as follows:

$$
A=\left[\begin{array}{ll}
R_{1} & S_{1} \\
T_{1} & P_{1}
\end{array}\right], B=\left[\begin{array}{ll}
R_{2} & S_{2} \\
T_{2} & P_{2}
\end{array}\right]
$$

such that $2 R_{i}>T_{i}+S_{i}$, and $T_{i}>R_{i}>P_{i}>S_{i}$, where $i=1$, 2. The dynamical equations for the spatial asymmetric prisoner's dilemma game (spatial APD) are:

$$
\begin{gathered}
\frac{\partial u_{1}}{\partial t}=D_{1} \nabla^{2} u_{1}+ \\
u_{1}\left(1-u_{1}\right)\left[-\left(P_{1}-S_{1}\right)+u_{2}\left(P_{1}-S_{1}-T_{1}+R_{1}\right)\right], \\
\frac{\partial u_{2}}{\partial t}=D_{2} \nabla^{2} u_{2}+ \\
u_{2}\left(1-u_{2}\right)\left[-\left(P_{2}-S_{2}\right)+u_{1}\left(P_{2}-S_{2}-T_{2}+R_{2}\right)\right],
\end{gathered}
$$

where $u_{1}\left(u_{2}\right)$ is the fraction of cooperators in the first (second) players population. The solution $u_{1}=u_{2}=0$ is linearly asymptotically stable. It represents the always defect strategy.

Two questions arise the first is: can diffusion stabilize the cooperation solution $u_{1}=u_{2}=1$ ? And does the always defect solution have (FAI)

nonlinear instability? Using the techniques of the previous section we get:

\section{Proposition (4):}

(i) If $D_{i} \pi^{2}>T_{i}-R_{i}$, then the cooperation solution is linearly asymptotically stable.

(ii) The always defect solution does not have FAI.

\section{Telegraph reaction diffusion in spatial asym- metric games}

The standard spatial games depend on the familiar reaction-diffusion equation

$$
\frac{\partial u(x, t)}{\partial t}=D \frac{\partial^{2} u(x, t)}{\partial x^{2}}+f(u) .
$$


A basic weakness in this equation is that the flux $j$ reacts simultaneously to the gradient of $u$ consequently an unbounded propagation speed is allowed. This manifests itself in many solutions to Eq. (1) e.g. (if $f=0$ ), then

$$
u(x, t)=\frac{1}{\sqrt{4 \pi D t}} e^{\frac{-x^{2}}{4 D t}}, u(x, 0)=\delta(x) \text { i.e } u(x, t)>0 \forall x, \forall t>0 .
$$

This is unrealistic specially in biological and economical systems, where it is known that propagation speeds are typically small. To rectify this weakness, Fick's law is replaced by

$$
j+\tau \frac{\partial j}{\partial t}=D \frac{\partial u}{\partial x}
$$

and the resulting telegraph diffusion equation becomes

$$
\tau \frac{\partial^{2} u}{\partial t^{2}}+\frac{\partial u}{\partial t}=D \frac{\partial^{2} u}{\partial x^{2}}
$$

The corresponding telegraph reaction diffusion (TRD) is

$$
\tau \frac{\partial^{2} u}{\partial t^{2}}+\left(1-\tau \frac{\mathrm{d} f}{\mathrm{~d} u}\right) \frac{\partial u}{\partial t}=D \nabla^{2} u+f(u)
$$

The time constant $\tau$ can be related to the memory effect of the flux $j$ as a function of the distribution $u$ as follows: Assume that [Compte and Metzle 1997]

$$
j(x, t)=-\int_{0}^{t} K\left(t-t^{\prime}\right) \frac{\partial u\left(x, t^{\prime}\right)}{\partial x} \mathrm{~d} t^{\prime}
$$

hence

$$
j+\tau \frac{\partial j}{\partial t}=-\tau K(0) u(x, t)-\int_{0}^{t}\left(\tau \frac{\partial K\left(t-t^{\prime}\right)}{\partial t}+K\left(t-t^{\prime}\right)\right) \frac{\partial u}{\partial x} \mathrm{~d} t^{\prime} .
$$

This equation is equivalent to the telegraph equation if

$$
K(t)=\frac{D}{\tau} \exp \left(\frac{-t}{\tau}\right) .
$$

This lends further support that TRD is more suitable for economic and biological systems than the ordinary diffusion equation since e.g. it is known that we take our decisions according to our previous experiences, so memory effects are quite relevant. Further evidence comes from the work of Chopard 
and Droz [Chopard and Droz 1991], where they have shown that starting from discrete time and space then the continuum limit does not give the standard reaction diffusion but the telegraph one.

Since it is known that new technologies, habits etc... takes time to spread, we believe that TRD equation is more relevant than the ordinary diffusion equation in modelling economic and biological systems [Ahmed et al 2001].

Now we apply TRD to spatial APD game. The TRD for a system of equations are [Hadeler 1998]

$$
\begin{gathered}
\tau \frac{\partial^{2} u_{i}}{\partial t^{2}}+\frac{\partial u_{i}}{\partial t}-\tau \sum_{j} \frac{\partial u_{j}}{\partial t} \frac{\partial f_{i}}{\partial u_{j}}= \\
D \nabla^{2} u_{i}+f_{i}\left(u_{1}, u_{2}, \ldots, u_{n}\right),
\end{gathered}
$$

hence applying it to the APD (18), we get

$$
\begin{gathered}
\tau \frac{\partial^{2} u_{1}}{\partial t^{2}}+\frac{\partial u_{1}}{\partial t}-\tau \frac{\partial u_{1}}{\partial t}\left(1-2 u_{1}\right)\left[-\left(P_{1}-S_{1}\right)+\right. \\
\left.u_{2}\left(P_{1}-S_{1}-T_{1}+R_{1}\right)\right]=D_{1} \nabla^{2} u_{1}+ \\
u_{1}\left(1-u_{1}\right)\left[-\left(P_{1}-S_{1}\right)+u_{2}\left(P_{1}-S_{1}-T_{1}+R_{1}\right)\right], \\
\tau \frac{\partial^{2} u_{2}}{\partial t^{2}}+\frac{\partial u_{2}}{\partial t}-\tau \frac{\partial u_{2}}{\partial t}\left(1-2 u_{2}\right)\left[-\left(P_{2}-S_{2}\right)+\right. \\
\left.u_{1}\left(P_{2}-S_{2}-T_{2}+R_{2}\right)\right]=D_{2} \nabla^{2} u_{2}+ \\
u_{2}\left(1-u_{2}\right)\left[-\left(P_{2}-S_{2}\right)+u_{1}\left(P_{2}-S_{2}-T_{2}+R_{2}\right)\right] .
\end{gathered}
$$

The following question arises: Can diffusion stabilize the cooperation solution $u_{1}=u_{2}=1$ ? Using the techniques of the second section, we get

Proposition (5): If the following conditions are satisfied

$$
\begin{gathered}
D_{i} \pi^{2}>T_{i}-R_{i}, 1>\tau\left(T_{i}-R_{i}\right), \\
4 \tau\left(D_{i} \pi^{2}-T_{i}+R_{i}\right) \leq\left[-1+\tau\left(T_{i}-R_{i}\right)\right]^{2}, i=1,2,
\end{gathered}
$$

then the cooperation solution is linearly asymptotically stable.

Proof. Assume that

$$
u_{1}=1-\varepsilon_{1} \exp \left(\sigma_{1} t\right) \sin (\pi x), u_{2}=1-\varepsilon_{2} \exp \left(\sigma_{2} t\right) \sin (\pi x) .
$$

Substituting one gets

$$
\begin{gathered}
\sigma_{1}=\frac{1}{2 \tau}\left[\left(-1+\tau\left(T_{1}-R_{1}\right)\right) \pm\right. \\
\left.\sqrt{\left(-1+\tau\left(T_{1}-R_{1}\right)\right)^{2}-4 \tau\left(R_{1}-T_{1}+D_{1} \pi^{2}\right)}\right] \\
\sigma_{2}=\frac{1}{2 \tau}\left[\left(-1+\tau\left(T_{2}-R_{2}\right)\right) \pm\right. \\
\left.\sqrt{\left(-1+\tau\left(T_{2}-R_{2}\right)\right)^{2}-4 \tau\left(R_{2}-T_{2}+D_{2} \pi^{2}\right)}\right]
\end{gathered}
$$


Stability requires that the real part of $\sigma_{i}, i=1,2$ is negative. The first two conditions in the proposition guarantee this requirement. Furthermore since $u_{i}, i=1,2$ are real and nonnegative, then the term under the square root should be nonnegative. The third condition of the proposition guarantees $u_{i} \geq 0$.

Thus the conditions for cooperation stability for TRD are more stringent than those for ordinary diffusion (c.f. proposition (4)).

\section{$5 \quad$ Asymmetric game of parental investment}

Parents are faced with the decision whether to care for the offsprings or to desert. A model has been given for this asymmetric game [Krebs and Davies 2000]. Let $p_{0}, p_{1}, p_{2}$, be the probabilities of survival of offsprings which are not cared for, cared for by a single parent and cared for by both parents, respectively, then $p_{0}<p_{1}<p_{2}$. A deserting male has a chance $q$ of mating again while a caring (deserting) female has $w_{1}\left(w_{2}\right)$ offsprings. The payoff matrices for male (female) corresponding to the strategies $\mathrm{C}$ (care) or $\mathrm{D}$ (desert) are denoted by $A(B)$, and given by

$$
A=\left[\begin{array}{ll}
w_{1} p_{2} & w_{2} p_{1} \\
w_{1} p_{1}(1+q) & w_{2} p_{0}(1+q)
\end{array}\right], \quad B=\left[\begin{array}{ll}
w_{1} p_{2} & w_{1} p_{1} \\
w_{2} p_{1} & w_{2} p_{0}
\end{array}\right] .
$$

The spatial asymmetric equations for the above game are:

$$
\begin{gathered}
\frac{\partial u}{\partial t}=u\left[w_{1} p_{2} v+w_{2} p_{1}(1-v)-w_{1} p_{2} u v-w_{2} p_{1} u(1-v)-\right. \\
\left.w_{1} p_{1}(1+q) v(1-u)-w_{2} p_{0}(1+q)(1-u)(1-v)\right]+D_{1} \frac{\partial^{2} u}{\partial x^{2}}+D_{12} \frac{\partial^{2} v}{\partial x^{2}}, \\
\frac{\partial v}{\partial t}=v\left[w_{1} p_{2} u+w_{1} p_{2}(1-u)-w_{1} p_{2} u v-w_{2} p_{1} u(1-v)-\right. \\
\left.w_{1} p_{1} v(1-u)-w_{2} p_{0}(1-u)(1-v)\right]+D_{2} \frac{\partial^{2} v}{\partial x^{2}}+D_{21} \frac{\partial^{2} u}{\partial x^{2}} .
\end{gathered}
$$

In this system, we introduced both ordinary and cross diffusion. Cross diffusion is the diffusion of one type of species due to the presence of another [Okubo 1980]. This phenomena is abundant in nature e.g. predator-prey systems where the predator diffuses towards the regions where the prey is more abundant. On the other hand the prey tries to avoid predators by diffusing away from it. Another area of application is in epidemics where susceptible individuals try to avoid infected ones.

Here we will see that ordinary diffusion is unable of destabilizing the diffusionless ESS: 
(i) ESS1 where both male and female desert i.e. $(u=0, v=0)$. It requires $w_{2} p_{0}>w_{1} p_{1}$ and $p_{0}(1+q)>p_{1}$.

(ii) ESS2 where male cares and female desert i.e. $(u=1, v=0)$. It requires $w_{2} p_{1}>w_{1} p_{2}$ and $p_{0}(1+q)<p_{1}$.

(iii) ESS3 where female cares and male desert i.e. $(u=0, v=1)$. It requires $w_{1} p_{1}>w_{2} p_{0}$ and $p_{1}(1+q)>p_{2}$.

(iv) ESS4 where both male and female care i.e. $(u=1, v=1)$. It requires $w_{1} p_{2}>w_{2} p_{1}$ and $p_{1}(1+q)<p_{2}$.

Following steps similar to the previous games, we get

Proposition (6): The solution ESSi, $i=1,2,3,4$ is destabilized if the following condition is satisfied:

$$
D_{12} D_{21} \pi^{4}>\left(D_{1} \pi^{2}-a_{i}\right)\left(D_{2} \pi^{2}-b_{i}\right)
$$

where

$$
\begin{aligned}
& a_{1}=w_{2} p_{1}-w_{2} p_{0}(1+q), \quad b_{1}=w_{1} p_{1}-w_{2} p_{0}, \\
& a_{2}=-w_{2} p_{1}+w_{2} p_{0}(1+q), \quad b_{1}=w_{1} p_{2}-w_{2} p_{1} \\
& a_{3}=w_{1} p_{2}-w_{1} p_{1}(1+q), \quad b_{1}=-w_{1} p_{1}+w_{2} p_{0} \\
& a_{4}=-w_{1} p_{2}+w_{1} p_{1}(1+q), \quad b_{1}=w_{2} p_{1}-w_{1} p_{2} .
\end{aligned}
$$

Notice that all $a_{i}, b_{i}, i=1,2,3,4$ are negative hence ordinary diffusion cannot destabilize the ESS.

Applying the above procedure to the battle of the sexes [Schuster and Sigmund 1981] where the female has two strategies coy or fast while the male can be either faithful or philanderer. The male (female) payoff matrix is $A(B)$

$$
A=\left[\begin{array}{ll}
0 & -10 \\
-2 & 0
\end{array}\right], \quad B=\left[\begin{array}{ll}
0 & 5 \\
3 & 0
\end{array}\right],
$$

hence the spatial battle of the sexes equations are

$$
\begin{gathered}
\frac{\partial u}{\partial t}=u(1-u)(-10+12 v)+D_{1} \frac{\partial^{2} u}{\partial x^{2}} \\
\frac{\partial v}{\partial t}=v(1-v)(5-8 u)+D_{2} \frac{\partial^{2} v}{\partial x^{2}} .
\end{gathered}
$$


Proposition (7): Diffusion stabilizes the internal equilibrium of the system (27).

Proof. There is a unique internal homogeneous equilibrium solution $E=$ $(5 / 8,5 / 6)$, which (for the diffusionless case) is stable but not asymptotically stable. Substituting with

$$
u=\frac{5}{8}+\varepsilon \exp (\sigma t) \sin \pi x, \quad v=\frac{5}{6}+\varsigma \exp (\sigma t) \sin \pi x, 1 \geq x \geq 0,
$$

in Eq. (27), and linearizing in $\varepsilon, \zeta$, one gets

$$
\left(\sigma+D_{1} \pi^{2}\right) \varepsilon=\frac{45}{16} \zeta,\left(\sigma+D_{2} \pi^{2}\right) \zeta=-\frac{10}{9} \varepsilon .
$$

Hence

$$
\left[\left(\sigma+D_{1} \pi^{2}\right)\left(\sigma+D_{2} \pi^{2}\right)+\frac{25}{8}\right] \zeta=0,
$$

i.e.

$$
\left(\sigma+D_{1} \pi^{2}\right)\left(\sigma+D_{2} \pi^{2}\right)+\frac{25}{8}=0,
$$

then

$$
\sigma^{2}+\sigma\left(D_{1} \pi^{2}+D_{2} \pi^{2}\right)+\left(\frac{25}{8}+D_{1} D_{2} \pi^{4}\right)=0 .
$$

Therefore the real part of $\sigma$ is negative, then the internal equilibrium (in the presence of diffusion) is asymptotically stable. It is clear that if the diffusion coefficients are set equal to zero $\left(D_{1}=D_{2}=0\right)$, then one regains the stability but not asymptotic stability. This completes the proof.

\section{Conclusions}

Based on replicator equations, a mathematical approach for the analysis of spatial asymmetric games is introduced. Some questions regarding spatial stability for asymmetric hawk-dove and the asymmetric prisoner's dilemma (APD) games are answered. Telegraph reaction diffusion equation is applied for stability analysis of the asymmetric prisoner's dilemma game. Asymmetric game of parental investment is studied in the presence of both ordinary 
and cross diffusions. Ordinary diffusion cannot destabilize the ESS for this game. Conditions for destabilizing the ESS are given in the case of cross diffusion.

\section{Acknowledgments}

We thank the referees for their helpful comments.

\section{References}

Ahmed E., Abdusalam H. A. and Fahmy I. (2001), Telegraph reaction diffusion equations. Int. J. Mod. Phys. C 12, 717-726.

Boerlijst M. C. and Hogeweg P. (1995), Attractors and spatial patterns in hypercycles with negative interactions. J. Theor. Biol. 176, 199-210.

Chopard B. and Droz M. (1991), Cellular automata model for the diffusion equation, J. Stat. Phys. 64, 859-892.

Compte A. and Metzle R. (1997), The generalized Cattaneo equation for the description of anomalous transport processes. J. Phys. A 30, 7277-7289.

Cressman R. and Vickers G. T. (1997), Spatial and density effects in evolutionary game theory. J. Theor. Biol. 184, 359-369.

Hadeler K. P. (1998). in O. Diekman et al (eds), Mathematics inspired by Biology. Springer, Berlin.

Hofbauer J., Hutson V. and Vickers G. T. (1997), Travelling waves for games in economics and biology. Nonlinear Analysis, Theory. Methods and Applications 30, 1235-1244.

Hofbauer J. and Sigmund K. (1998), Evolutionary games and population dynamics. Cambridge University Press, Cambridge.

Krebs J. R. and Davies N. B. (2000), An introduction to behavioral ecology. Blakwell scientific pub., Oxford.

Okubo A. (1980), Diffusion and ecological problems. Springer, Berlin. 
Schuster P. and Sigmund K. (1981), Coyness philandering and stable strategies. Anim. Behav. 29, 186-192.

Stuart A. (1989), Nonlinear instability in dissipative finite difference scheme. Siam Rev. 31, 191-220. 